\title{
Effect of urban green patterns on surface urban cool islands and its seasonal variations
}

\author{
Ailian Chen ${ }^{\mathrm{a}, \mathrm{b}, *}, \mathrm{X}$. Angela Yao ${ }^{\mathrm{c}}$, Ranhao Sun ${ }^{\mathrm{a}}$, Liding Chen ${ }^{\mathrm{a}}$ \\ a State Key Lab of Urban and Regional Ecology, Research Center for Eco-Environmental Sciences, CAS, Beijing 100085, China \\ b University of Chinese Academy of Sciences, CAS, Beijing 100049, China \\ ' Department of Geography, University of Georgia, Athens, GA 30602, USA
}

\section{A R T I C L E I N F O}

\section{Keywords:}

Beijing

Landscape metrics

Landscape pattern

Land surface temperature

Urban heat island

\begin{abstract}
A B S T R A C T
Urban green spaces often form urban cool islands (UCIs), which are important for human health and urban sustainability. Previous studies have emphasized the cooling effects of urban green spaces on their surrounding areas at landscape level. Less attention, however, has been directed to effects of urban green space patterns on their own UCIs at patch level. In this study, we focused on the effects of spatial patterns of urban green patches on their own surface UCIs. The urban green spaces of Beijing, China, were extracted from one QuickBird image and were classified as Trees, Shrubs, Grass, Crops, River and Lake. Land surface temperatures (LSTs) were derived from four Landsat images, each in one season. The UCI was represented by the minimum LST of each urban green patch. Results showed spatial patterns of urban green patches had significant effects on their UCIs in four seasons. In detail, the size, edge and connectivity of urban green spaces all affected the UCIs negatively, and the influence was stronger in warm seasons. Shape of urban green space also had effects on UCIs, but the effects were stronger in cool seasons. Great differences were found between predictive values of metrics for different green types. Shape metrics were more important for indicating UCIs of River, Trees and Crops than were patch size and connectivity. However, patch size and connectivity metrics were more effective in determining UCIs of Shrubs, Grass and Lake than were shape metrics. Further, among shape metrics, only shape index was a good indicator of UCIs. The results of this study suggest that a combination of specific urban green types and pattern metrics are a prerequisite for analyzing the influence of urban green patterns on UCIs and for urban green design.
\end{abstract}

(C) 2014 Elsevier GmbH. All rights reserved.

\section{Introduction}

Urbanization has resulted in intensive concentrations of population, high buildings and impervious pavements. It exacerbates the urban climate environment characterized by the urban heat island (UHI) effect, although it provides convenient living and access to educational and medical facilities. Deterioration of the urban climate is consequently associated with heat waves (Fischer et al., 2007), disease and health risk to more than $50 \%$ of the world's population (Frumkin and McMichael, 2008). Thus, UHI mitigation is urgent in order to improve urban living conditions (Opdam et al., 2009).

\footnotetext{
* Corresponding author at: State Key Lab of Urban and Regional Ecology, Research Center for Eco-Environmental Sciences, CAS, Beijing 100085, China. Tel.: +86 13466727394

E-mail addresses: cal-0601@163.com, alchen_0601@yahoo.com (A. Chen).
}

Urban green spaces, which refer to all kinds of green infrastructures that use vegetated spaces and bodies of water for the public well-being, form cool islands throughout warm seasons and help to regulate the urban microclimate (Benedict and McMahon, 2006; Zoulia et al., 2009; Kantzioura et al., 2012). Urban greening efforts from courtyard scale to urban scale have been proved successful in alleviating outdoor UHI effects and improving indoor comfort (Bruse and Fleer, 1998; Shashua-Bar et al., 2009; Zoulia et al., 2009; Oliveira et al., 2011; Susca et al., 2011; Mackey et al., 2012; Ng et al., 2012). For instance, an increase of grassland in parking lots brings a maximum decrease of $7.26^{\circ} \mathrm{C}$ to surface temperature in the summer (Onishi et al., 2010). This is because vegetated spaces transform a small part of the absorbed solar radiation by photosynthesis rather than convert that part to heat energy. Also, they do not become as warm as human constructed surfaces as a result of transpiration. Water bodies form cool islands mostly due to the high heat capacity. Besides, as the "nature" in the urban areas, urban green spaces are usually the outdoor "playgrounds", especially for 
very young and aged people (Oliveira et al., 2011). Therefore, the UCI effects of urban green spaces are increasingly emphasized in urban planning to mitigate UHIs and to improve urban human thermal comfort (Heidt and Neef, 2008; Hamada and Ohta, 2010; Harnik, 2010).

The UCIs refer to the phenomenon that green areas have lower temperature than that of impervious surface. Usually, there are two types of UCIs according to the data source used: atmospheric/air UCIs and surface UCIs. Air UCIs use air temperature from vehicletraverse or fixed weather station, and surface UCIs mostly use land surface temperature (LST) derived from thermal remote sensing images. Observations have shown that air UCIs exist in urban forests or grasslands throughout the day in almost all seasons except for winter when forest and grassland was warmer than built up areas. The air UCI intensity, which is usually the temperature difference between warm and cool areas, has been observed higher during the day than the night in summer and the opposite is true in winter (Cohen et al., 2012; Hamada and Ohta, 2010). Surface UCI intensity is also stronger during daytime than at night in almost all season, and is especially stronger in summer (Schwarz et al., 2011).

Air UCIs are important for human comfort, so are surface UCIs. Cohen et al. (2012) found a maximum air UCI intensity of $3.8^{\circ} \mathrm{C}$ in the summer that led to a $10^{\circ} \mathrm{C}$ decrease in mean radiant temperature (Tmrt). Tmrt is a primary indicator of human comfort and is sensitive to the surrounding radiative flux (Matzarakis et al., 2010), and thus to surface UCIs. Surface UCIs have the potentials to cool down the ambient air temperature by conduction and also indicate the relatively cooler radiative flux than built up areas. Surface UCIs can create a difference of more than $24^{\circ} \mathrm{C}$ between grass and concrete surface (e.g., Armson et al., 2012).

In studies of landscape ecology and urban planning, surface UCIs have been increasingly discussed from a parcel to regional scale (Cao et al., 2010; Sun et al., 2012), as it uses LST as data source. LST is the mean radiative temperature of an area of land surface, determined by the mean balance of solar heating, upward thermal radiation and convective heat loss to the air. It indicates the surface's ability and potential to radiate and conduct heat, which directly affects human comfort (Cohen et al., 2012). Besides, LST provides higher spatially continuous coverage and is easier to be related to remote sensed land cover maps than is air temperature (Voogt and Oke, 2003; Weng, 2009).

Previous studies regarding surface UCIs of urban green spaces focused either on one particular park (Oliveira et al., 2011), or on certain type of urban green space (e.g., bodies of water (Sun et al., 2012) or parks (Cao et al., 2010)). Few studies, however, have discussed the effect of urban green patterns on their own surface UCI effect, especially at patch level, to explore the effect of patch patterns on UCIs. Conversely, landscape-level studies were reported on the pattern effect of urban green (Li et al., 2012; Zhou et al., 2011). This is less applicable to implementation of urban green space design, which always starts from urban green patches. Nowadays, a challenge exists in arranging more urban green space in compact urbanized areas and every urban green space is important in providing ecosystem services. Therefore, it is necessary for green designing or urban planning to consider patches' patterns of urban greens, including the composition and the configuration at patch level.

Composition and configuration are the two aspects of spatial patterns. Composition is the amount of area and diversity of the types within a landscape. At patch level, composition is just the patch area. Configuration covers patch shape, edge and connectivity to neighborhood. The configuration of the landscape (e.g., shape, connectivity) has already been proven important in most landscape processes (such as soil erosion, Chen et al., 2009). In the process of urban climatization, previous studies at landscape level but not patch level have also confirmed the importance of shape (Sun et al.,
2012) or general landscape configuration (Zhou et al., 2011; Li et al., 2012).

This study thus used part of metropolitan Beijing as a case study and analyzed the effect of spatial patterns of urban green space on surface UCIs. The objectives were (1) to determine the effect of urban green patterns, especially configuration on UCIs and its seasonal variations, and (2) to identify spatial pattern indicators of each type of urban greens for surface UCI prediction.

\section{Methodology}

\section{Study area}

Beijing is located in north China at $39.9^{\circ} \mathrm{N}, 116.3^{\circ} \mathrm{E}$, and has a monsoon-influenced humid continental climate characterized by hot-humid summers and cold-windy-dry winters. The average daytime high temperature in January (usually the coolest month) is around $1{ }^{\circ} \mathrm{C}$, whereas that in July (usually the warmest month) is above $30^{\circ} \mathrm{C}$. Surface UHI analysis has shown great UHI intensity, ranging from 5 to $10^{\circ} \mathrm{C}$ (Wang et al., 2007).

As shown in Fig. 1, the transportation system in Beijing is characterized by a standardized network with 5 ring roads, comprised of the 2nd to 6th Ring Road successively from downtown to the suburbs. (Names of roads and some places of interests are labeled in Fig. 1 for the convenience of explaining the results.)

\section{Materials}

One QuickBird (QB) image taken on July 5, 2002, with 4 multispectral channels (resolution, $\sim 2.52$ by $2.53 \mathrm{~m}$ ) and 1 panchromatic channel (resolution, $\sim 0.63 \mathrm{~m}$,) were used for urban green space mapping. Four cloud-free Landsat ETM+ images were selected to retrieve LSTs. The "Landsat" satellites pass the study area at around 10:45, local time. The red channel (resolution, $30 \mathrm{~m}$ ), near-infrared channel (resolution, $30 \mathrm{~m}$ ) and thermal channel (resolution, $60 \mathrm{~m}$ ) of ETM+ images were used for LST retrieval. Acquisition dates of ETM+ images were May 22, July 9, October 13 and November 14. May 22 represented spring, the dry and warm season. October13 and November 14 represented autumn and winter, the cooler or cold and the dry seasons, respectively. July 9 represented summer, the hot and humid season. There was no rain or high wind for at least 4 days before the date of LST acquisition. The mean wind velocity is less than $2 \mathrm{~m} \mathrm{~s}^{-1}$ for the retrieval date, with no precipitation. These dates are no more than 4 months from acquisition date of the QB image that was used for urban green mapping, and we could thus assume that there was no significant change in urban green areas.

We chose images from 2002 because there were more seasonal Landsat images available than in other years. These images were all geometrically rectified and registered to the same projection system (Datum: WGS 84, UTM Zone North 50) in the professional image processing and analysis software - ENVI ${ }^{\mathrm{TM}}$ (Version 5.0). Channel 6 of the ETM+ data was resampled to a resolution of $30 \mathrm{~m}$ as the multi-spectral channel for retrieving LST.

\section{Urban green mapping}

Six types of urban green spaces were mapped visually: woodland (Trees), shrub land (Shrubs), grassland (Grass), cropland (Crops), water I (River) and water II (Lake), as defined in Table 1. We first delineated the boundary of each green patch manually on the QB channel 3, 4, 2-composite image (hereafter, QB composite image). We then determined and verified the types and edges of each patch on the fused image (fused with QB composite channels and pan channel, referred to hereafter as QB fused image). 


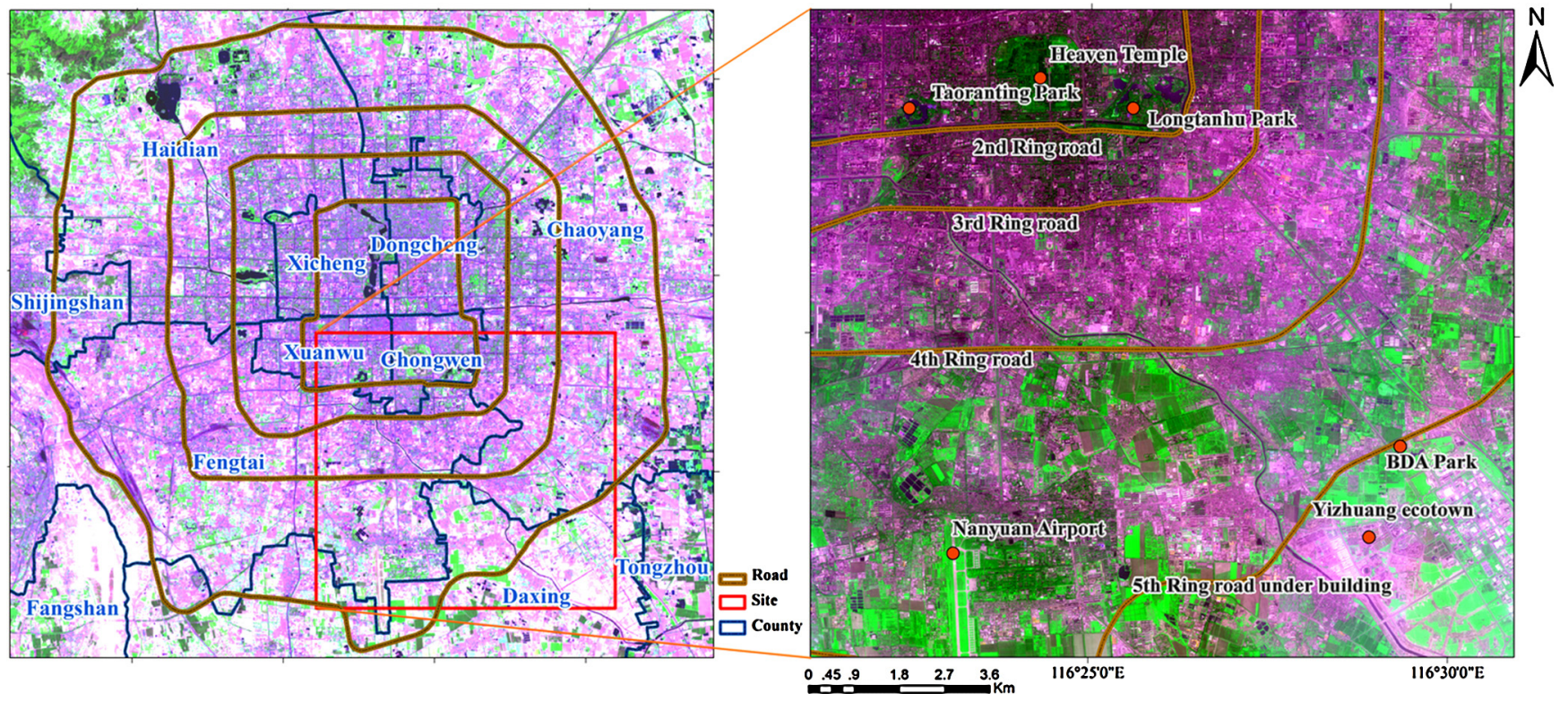

Fig. 1. (A) Location and (B) QuickBird image of the study area.

Table 1

Urban green types and implications.

\begin{tabular}{|c|c|c|}
\hline ID & Types (Common name) & Implication \\
\hline 1 & Woodland (Trees) & Green land dominated by woodland. Other vegetation less than $10 \%$ \\
\hline 2 & Shrub land (Shrubs) & Green land dominated by shrubs. Other vegetation less than $10 \%$ \\
\hline 3 & Grassland (Grass) & Human-grown grassland, lawn, with woodland or shrubs less than $10 \%$ \\
\hline 4 & Water I (River) & Stream, channel, river, and other running water \\
\hline 5 & Water II (Lake) & Lakes and relatively large, stable, open bodies of water \\
\hline 6 & Cropland (Crops) & Farm with green-up vegetation or crop field \\
\hline
\end{tabular}

Verification for our extraction was conducted in two steps. First, we assigned a unique color for each type for the vector layer with a transparency of $50 \%$ and overlaid this colored layer on the QB fused image as a background. Second, we corrected patches that had the same background but different patch colors.

\section{Urban green pattern metrics}

We chose 5 patch metrics from the metrics advanced in 1994 by McGarigal et al. (2012) and defined another 2 metrics using buffer analysis (Cao et al., 2010; Sun et al., 2012) to count the neighboring green area and proportion to describe urban green patterns, as shown in Table 2. There are three levels of pattern metrics, i.e., patch level, class (type) level and landscape level. We used patch level metrics for statistical analysis because we were dealing with the UCI of each green patch.

Patch area (PA) is a composition metric, which shows the size of each urban green patch, and is also a prescriptive quantity used by decision makers. Shape index (SI), Fractal dimension (FD), and perimeter-area ratio (PAR) are shape metrics, the performance of which was compared. SI refers to the shape of a circle. SI approaches 1 for a patch with simple circle shape. Higher SI indicates higher shape complexity. Similarly, FD describes the fractality or complexity of a patch referring to a square, and it "approaches 1 for shapes with very simple perimeters such as squares, and approaches 2 for shapes with highly convoluted, plane-filling" (having many twists and turns on the edge) perimeters (McGarigal et al., 2012). PAR is simply the ratio of perimeter to area. TE is an edge metric, which equals the perimeter of a patch at patch level. These shape and edge metrics all represent urban green configurations. These five metrics were counted in the Patch Analyst software developed by Rempel et al. (2012) and embedded into ArcGIS TM (Version 10.0).
Neighborhood green proportion (NGP) and neighborhood green area (NGA) were used as candidates for connectivity metrics, another kind of configuration. They were calculated by buffer analysis with a radius of $50 \mathrm{~m}$ in ArcGIS ${ }^{\mathrm{TM}}$ software, but not in Patch Analyst, because we counted all the metrics directly from the vector layer, for which Patch Analyst provides no tools to calculate neighborhood connectivity.

\section{Land surface temperature and surface urban cool island}

LST was retrieved from thermal channels of ETM+ images mainly according to the Planck Law. This was done according to the following 3 steps: (a) the image digital number was converted back to at-sensor radiance with the scaling parameters provided along with the image metafile (NASA, 2000), (b) at-sensor radiance was converted to at-sensor temperature according to the Planck Law in Eqs. (1) and (2), and (c) the at-sensor temperature was adjusted to LST with land surface emissivity $(\varepsilon$ ) by Eq. (3).

The Planck Law is shown in Eq. (1).

$M_{\lambda}(T)=\frac{2 \pi h c^{2} \lambda^{-5}}{\left[e^{(h c / l k T)}-1\right]}$

In our retrieval, $M_{\lambda}(T)$ is the at-sensor radiance of Channel 6 with a wavelength of $\lambda ; h$ is the Planck constant, which equals $6.626 \times 10^{-34} \mathrm{Js} ; K$ is the Boltzmann constant, which equals $1.3806 \times 10^{-23} \mathrm{JK}^{-1}$; $c$ is the velocity of light, which is $2.998 \times 10^{8} \mathrm{~m} \mathrm{~s}^{-1}$; and $T$ (unit, $\mathrm{K}$ ) is the thermodynamic/Kelvin temperature. With these constants and the Landsat 7 Science Data Users Handbook (NASA, 2000), T can be retrieved by Eq. (2).

$T=\frac{1282.71}{\ln \left(666.09 / M_{\lambda}(T)+1\right)}$ 
Table 2

Pattern metrics used to describe urban green patterns.

\begin{tabular}{|c|c|c|}
\hline Metrics (unit) & Abbreviation & Equations (metric range) \\
\hline Patch Area ( $\mathrm{m}^{2}$ or hectare) & PA & $(\mathrm{PA}>0)$ \\
\hline Shape index & SI & $\mathrm{SI}=\mathrm{P} /(2 \sqrt{ } \pi \mathrm{PA}),(\mathrm{SI} \geq 1)$ \\
\hline Perimeter area ratio & PAR & $\mathrm{PAR}=\mathrm{P} / \mathrm{PA}(\mathrm{PAR}>0)$ \\
\hline Total edge $(\mathrm{m})$ & TE & $\mathrm{TE}=$ Perimeter, for a patch $(\mathrm{TE}>0) \mathrm{TE}=$ Sum of patch perimeters of the same type or class, for a class $(\mathrm{TE}>0)$ \\
\hline Fractal dimension & FD & $\mathrm{FD}=2 \ln (0.25 \mathrm{P}) / \ln (\mathrm{PA})(1 \leq \mathrm{FD} \leq 2)$ \\
\hline Neighborhood green area & NGA & NGA $=$ Sum of area of urban green that lies within the 50-meter buffer zone of each patch \\
\hline Neighborhood green proportion & NGP & $\mathrm{NGP}=$ urban green lying within the 50 -meter buffer zone of each patch/the area of that buffer zone \\
\hline
\end{tabular}

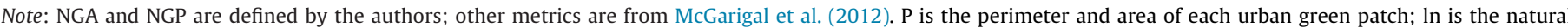
logarithm.

This at-sensor temperature was corrected to LST with land surface emissivity by Eq. (3).

$T=\frac{1282.71}{\ln \left(666.09 /\left(\varepsilon M_{\lambda}(T)\right)+1\right)}$

where $\varepsilon$ is the land surface emissivity estimated by the Normalized Difference Vegetation Index (NDVI) according to the method detailed by Sobrino and Raissouni (2000) and Sobrino et al. (2004). NDVI was calculated by Eq. (4).

$N D V I=\frac{\rho(\text { Channel } 4)-\rho(\text { Channel } 3)}{\rho(\text { Channel } 4)+\rho(\text { Channel } 3)}$

where $\rho$ (Channel4) and $\rho$ (Channel3) are top-of-atmosphere (TOA) reflectance transformed from the digital number of ETM+ Channels 4 and 3, respectively. TOA reflectance is the at-sensor reflectance with the cosine effect of the solar zenith and the exo-atmospheric solar irradiance due to variation in the sun-earth distance removed (Chander and Groeneveld, 2009; Li et al., 2011).

We neglected the atmospheric effect on the retrieval result as $\mathrm{Li}$ et al. (2011) did, based on three reasons. (1) Atmospheric correction based on an image or model with general parameters resulted in little error for the LST retrieval (Sobrino et al., 2004), (2) the images were generally cloud free, and (3) the comparison in our study was between the correlation coefficients and not based on the absolute LST value itself. Thus, the atmospheric effect on LST throughout one image was considered consistent and negligible.

The resulting LST in units Kelvin (K) was finally converted to Celsius $\left({ }^{\circ} \mathrm{C}\right)$ units, and the surface $\mathrm{UCI}$ was represented by the minimum LST of each urban green patch.

\section{Statistical analysis}

Statistical analyses were performed using the SPSS ${ }^{\mathrm{TM}}$ software. First, bivariate correlations were carried out between pattern metrics of each green type and UCIs of the four dates to determine seasonal variations in the effect of urban green patterns on UCIs. Spearman's rho as the correlation coefficient was used because the source data for pattern metrics are category classification data (Sun and Chen, 2012). Second, in considering that correlations might also exist among the metrics, stepwise regression analyses were conducted for each specific green type with the UCIs of summer to determine good metric indicators for UCIs as UCI effect is usually more important in the summer than in other seasons.

\section{Results}

Spatial pattern of urban green infrastructures and LST

\section{Spatial pattern of urban green infrastructures}

The total area of urban green infrastructure was about 6450 hectares, covering $35 \%$ of the overall study area. Specifically, the $35 \%$ urban green infrastructure was composed of $14 \%$ cropland, $12 \%$ woodland, $5 \%$ grassland, $2 \%$ shrubs, and $2 \%$ water. The distribution of urban green is shown in Fig. 2.

\section{Spatial pattern of LST}

The distribution of LST and UCIs corresponded to the pattern of urban green space, as shown in Fig. 3. The regions around the Heaven Temple, Taoranting Park, and Longtanhu Park formed the largest cool islands in all 4 seasons. Some agricultural land between the 4th and 5th Ring Roads also formed large cool islands in May, July and October. River showed an obvious cool corridor in May and July, but the cooling effect was less strong in October and November.

The highest maximum LST and the biggest temperature difference (TD) of the four dates occurred on May 22. Compared to May 22 , other dates all had lower maximum LSTs and lower TDs, indicating that May 22 had most heterogeneous thermal environment. The average UCIs formed by each green type were cooler than the mean temperature of the overall study area in all dates except for Grass, River and Crops on November 14, as shown in Table 3. This was because, crops had been harvested, grass withered earlier than most trees and shrubs, and water in river shrank in November. Lakes formed the coolest islands in all four seasons followed by River or Trees. Trees were generally cooler than other vegetation types.

\section{Effects of urban green pattern on UCI}

\section{Seasonal variations}

UCIs formed by urban greens in the four seasons correlated significantly with all seven pattern metrics except SI, PAR and FD, which had inconsistent coefficients between vegetation and water. The correlations of patch size, edge and connectivity were stronger on July 9, whereas this was not the case for SI, as shown in Table 4. The correlation between metrics and UCI are detailed below.

Size, edge and connectivity metrics all showed negative correlations to UCI on all four dates, and the correlation was especially strong with the UCIs of July 9. The correlation coefficients of these metrics were also higher for vegetation UCIs than for water UCIs.

As for shape metrics, SI correlated significantly with vegetation UCIs on all 4 dates and most strongly on October 13 and November 14 when there is less green, whereas FD and PAR did not have correlations for vegetation UCIs on one of the four dates. PAR correlated positively with water UCIs on all dates and especially strongly with UCIs on July 9. SI and FD showed flickering effects on water UCIs.

NGA had the strongest effect on UCIs of all four dates except for November, compared to all other metrics.

\section{Effects of urban green types}

Stepwise regression analysis was conducted between UCIs of July 9 and the pattern metrics for the six specific green types. Results showed that the indicator values (standardized coefficients) of pattern metrics for the UCIs differed greatly among urban green types. For Trees, Crops and River, their shape and edge 


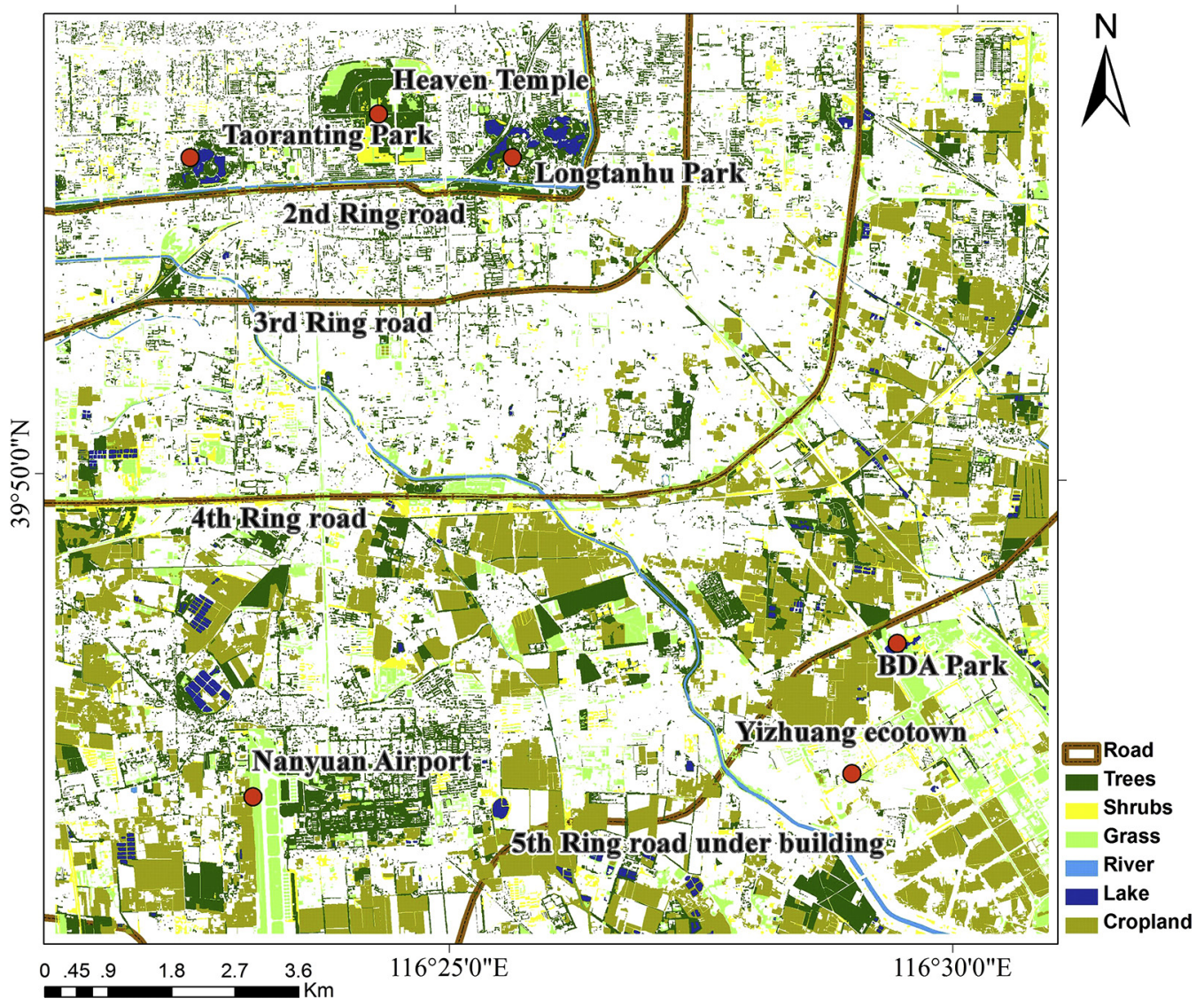

Fig. 2. Urban green spaces of the whole study area.

Table 3

Minimum (MIN) and Mean surface temperature of each green type.

\begin{tabular}{|c|c|c|c|c|c|c|c|c|}
\hline \multirow{2}{*}{$\begin{array}{l}\text { Dates } \\
\text { Temperature }\left({ }^{\circ} \mathrm{C}\right)\end{array}$} & \multicolumn{2}{|c|}{ May 22, 2002} & \multicolumn{2}{|c|}{ July 9, 2002} & \multicolumn{2}{|c|}{ October 13, 2002} & \multicolumn{2}{|c|}{ November 14, 2002} \\
\hline & Min & Mean & Min & Mean & Min & Mean & Min & Mean \\
\hline Study area & 23.18 & 38.17 & 20.49 & 28.98 & 9.85 & 19.52 & 0.00 & 9.69 \\
\hline Trees & 24.70 & 35.86 & 23.80 & 27.65 & 9.85 & 18.30 & 0.00 & 9.48 \\
\hline Shrubs & 26.19 & 37.45 & 25.22 & 28.28 & 15.01 & 19.07 & 4.41 & 9.66 \\
\hline Grass & 25.76 & 37.47 & 23.80 & 27.99 & 12.48 & 19.26 & 3.17 & 9.82 \\
\hline River & 29.32 & 33.72 & 25.22 & 27.08 & 15.28 & 18.61 & 6.06 & 9.75 \\
\hline Lake & 23.80 & 29.92 & 23.80 & 26.14 & 14.17 & 16.86 & 3.79 & 7.25 \\
\hline Crops & 25.33 & 37.24 & 23.28 & 27.69 & 11.02 & 18.91 & 0.63 & 10.08 \\
\hline
\end{tabular}

were more important than areas or neighborhood connectivity in determining their UCIs, but this was not the case for Shrubs, Grass and Lake. Details of regression models for urban green types (shown in Table 5) are as follows.
Trees: all metrics were included in the best fit model. The most important metrics were shape metrics. Orders of importance were SI, TE and FD. SI negatively influenced UCI (standardized coefficient $\beta=-1.41)$, whereas TE $(\beta=1.21)$ and $\operatorname{FD}(\beta=0.80)$ influenced

Table 4

Spearman correlation coefficients among urban green pattern metrics and UCIs of the four dates.

\begin{tabular}{|c|c|c|c|c|c|c|c|c|}
\hline \multicolumn{5}{|c|}{ Vegetation $(N=1089)$} & \multicolumn{4}{|c|}{ Water $(N=122)$} \\
\hline Metrics & UCI_0522 & UCI_0709 & UCI_1013 & UCI_1114 & UCI_0522 & UCI_0709 & UCI_1013 & UCI_1114 \\
\hline SI & $-0.28^{* *}$ & $-0.30^{* *}$ & $-0.31^{* *}$ & $-0.31^{* *}$ & & $-0.21^{*}$ & $-0.24^{* *}$ & \\
\hline PAR & $0.16^{* *}$ & $0.40^{* *}$ & $0.12^{* *}$ & & $0.54^{* *}$ & $0.47^{* *}$ & $0.42^{* *}$ & $0.46^{* *}$ \\
\hline FD & $-0.11^{* *}$ & & $-0.16^{* *}$ & $-0.22^{* *}$ & $0.28^{* *}$ & & & \\
\hline $\mathrm{TE}$ & $-0.39^{* *}$ & $-0.54^{* *}$ & $-0.39^{* * *}$ & $-0.31^{* *}$ & $-0.30^{* *}$ & $-0.35^{* *}$ & $-0.33^{* *}$ & $-0.29^{* *}$ \\
\hline PA & $-0.39^{* *}$ & $-\mathbf{0 . 6 2} 2^{* *}$ & $-0.37^{* *}$ & $-0.26^{* *}$ & $-0.34^{* *}$ & $-0.38^{* *}$ & $-0.34^{* *}$ & $-0.31^{* *}$ \\
\hline NGA & $-0.45^{* *}$ & $-0.64^{* *}$ & $-0.41^{* *}$ & $-0.25^{* *}$ & $-0.49^{* *}$ & $-0.63^{* *}$ & $-0.42^{* *}$ & $-0.29^{* *}$ \\
\hline NGP & $-0.26^{* *}$ & $-0.39^{* *}$ & $-0.22^{* *}$ & $-0.06^{*}$ & $-0.29^{* *}$ & $-0.31^{* *}$ & $-0.18^{*}$ & \\
\hline
\end{tabular}

Correlation coefficients higher than 0.5 are marked with bold values.

* Correlation is significant at the 0.05 level (2-tailed).

** Correlation is significant at the 0.01 level (2-tailed).

The digits $0522,0709,1013$, and 1114 after UCI here represent the dates May 22, July 9 , October 13 , and November 14 . $N$ is the number of samples. 

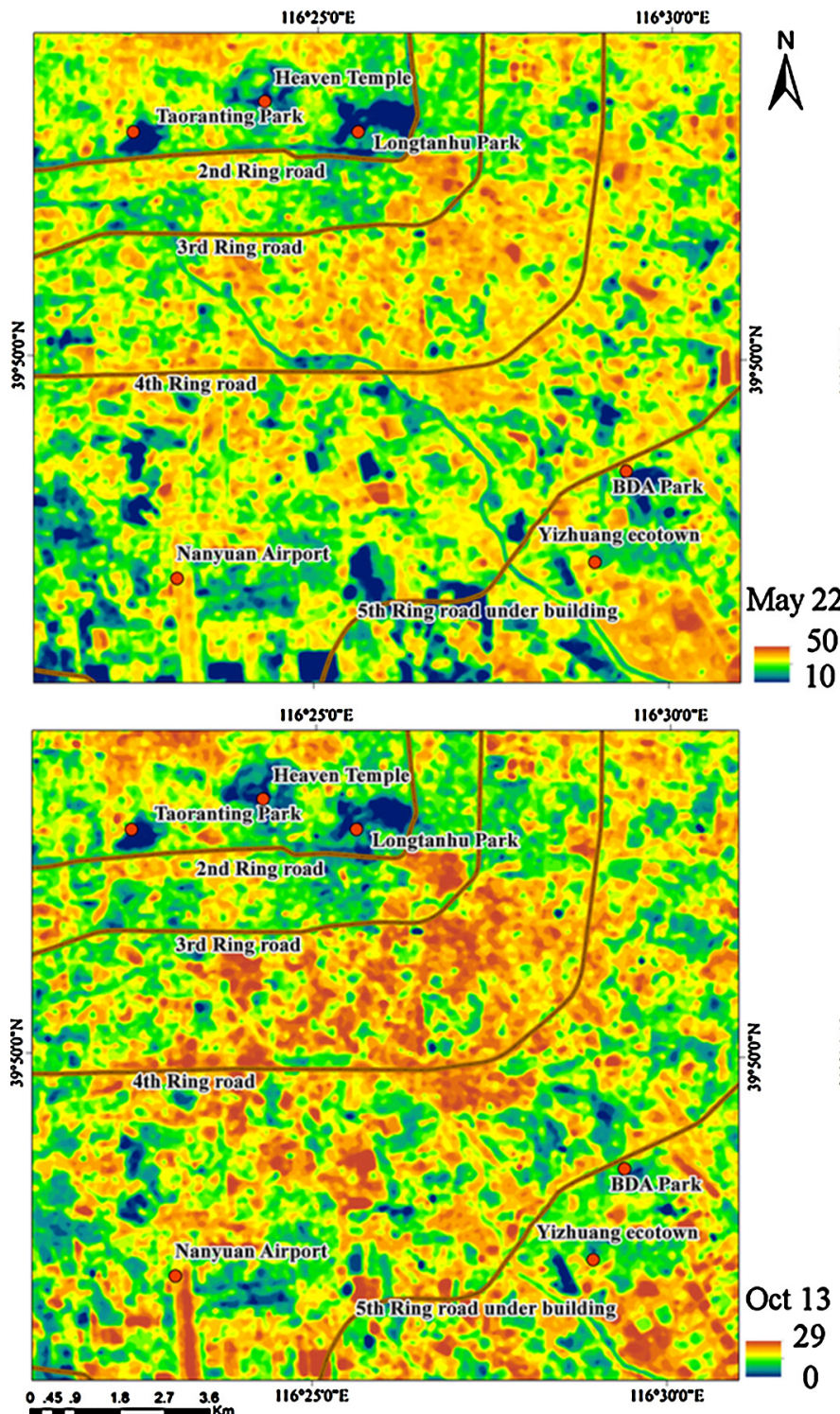

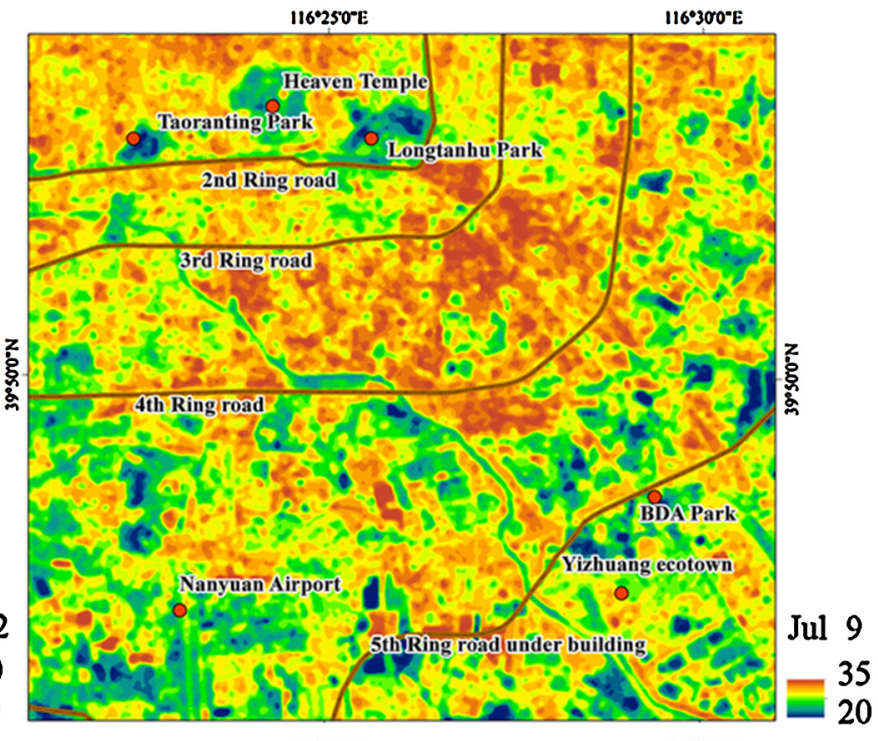

116.250'E

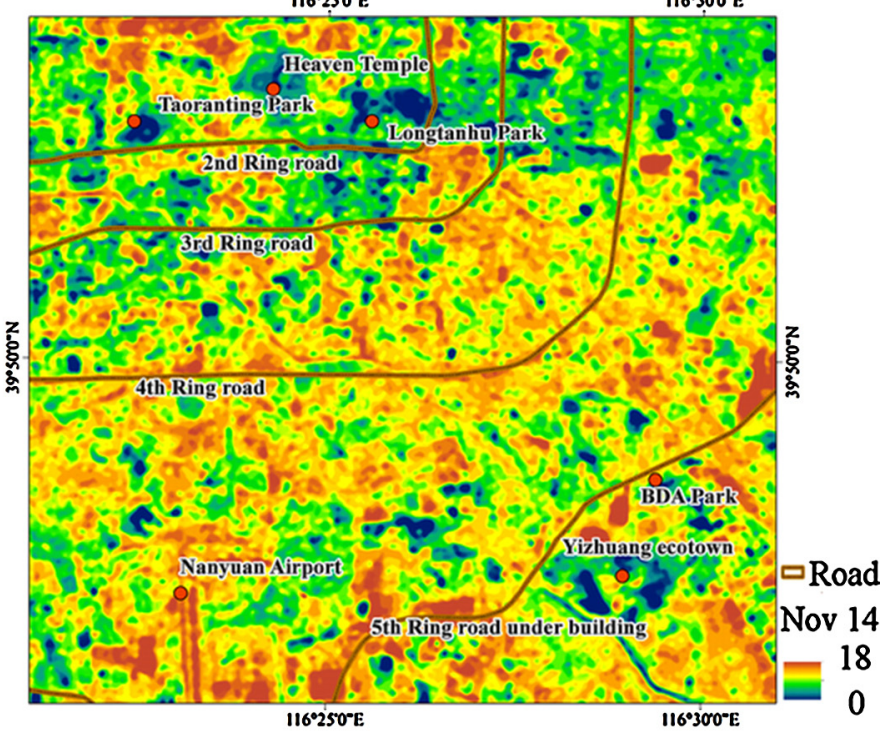

Fig. 3. Land surface temperature $\left({ }^{\circ} \mathrm{C}\right)$ of the four dates, May 22, July 9, October 13 and November 14 .

Table 5

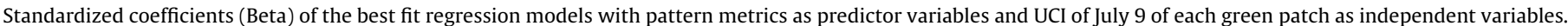

\begin{tabular}{|c|c|c|c|}
\hline Type $(N)$ & The best fit Model number (adjusted $R^{2}$ ) & Metrics entered & Standardized coefficients (Beta) \\
\hline \multirow[t]{6}{*}{ Trees $(N=256)$} & $6(0.44)$ & NGA & $-0.26^{* *}$ \\
\hline & & FD & $0.80^{* *}$ \\
\hline & & SI & $-1.41^{* *}$ \\
\hline & & $\mathrm{TE}$ & $1.21^{* *}$ \\
\hline & & PA & $-0.55^{* *}$ \\
\hline & & NGP & $-0.22^{* *}$ \\
\hline \multirow[t]{2}{*}{ Shrubs $(N=77)$} & $2(0.37)$ & PA & $-0.35^{* *}$ \\
\hline & & NGP & $-0.43^{* *}$ \\
\hline \multirow[t]{3}{*}{ Grass $(N=230)$} & $5(0.38)$ & SI & $-0.13^{*}$ \\
\hline & & PA & $-0.37^{* *}$ \\
\hline & & NGP & $-0.42^{* *}$ \\
\hline River $(N=18)$ & $1(0.51)$ & FD & $-0.74^{* *}$ \\
\hline Lake $(N=102)$ & $1(0.40)$ & NGA & $-0.64^{* *}$ \\
\hline \multirow[t]{5}{*}{ Crops $(N=522)$} & $7(0.40)$ & FD & $0.69^{* *}$ \\
\hline & & SI & $-1.24^{* *}$ \\
\hline & & PA & $-0.79^{* *}$ \\
\hline & & NGP & $-0.21^{* *}$ \\
\hline & & $\mathrm{TE}$ & $1.01^{* *}$ \\
\hline
\end{tabular}

\footnotetext{
$*$ Fitness is significant at the 0.05 level.
${ }_{*}^{*}$ Fitness is significant at the 0.01 level.
}

PA, TE, SI, FD, NGA, NGP are class area, total edge, shape index, neighborhood green area and proportion of urban green patch respectively. $N$ is the number of samples. 
UCI positively. After the three shape metrics, the most important metric was PA $(\beta=-0.55)$. Least important were the connectivity metrics, which influenced UCIs negatively ( $\beta$ of NGA $=-0.26, \beta$ of NGP $=-0.22$ ), just as PA did.

Shrubs: only PA and NGP were selected during stepwise regression. They both had negative impacts on UCI. NGP functioned more subtly compared to PA ( $\beta=-0.43$ for NGP versus $\beta=-0.35$ for PA).

Grass: NGP, PA, SI were the important factors for predicting $\mathrm{UCI}$, with NGP being the most important factor $(\beta=-0.42)$, much more so than PA $(\beta=-0.37)$ and $\mathrm{SI}(\beta=-0.13)$.

River and Lake: only one metric was included in the regression model for each of these water types. FD was for river, and NGA was for lake.

Crops: All metrics except NGA were selected in the best fit model. Similar to that for Trees, the most important predictive metric for Crops was SI, a shape metric $(\beta=-1.24)$, followed by the edge metric TE $(\beta=1.01)$, PA $(-0.79)$ and another shape metric, FD $(\beta=0.69)$.

\section{Discussion}

We used measurable metrics and quantitative statistical analysis to assess the relation between UCIs and urban green spatial patterns, which revealed patch size, shape, edge and connectivity all had effects on UCIs. We also found that the predictive value of pattern metrics for UCIs varied through the seasons and for green types. These results extend the scientific understanding of how urban green patterns influence UCIs. Meanwhile, they provide urban planners, landscape ecologists with some theoretical and methodological knowledge on urban green management, as well as on the use of landscape metrics.

\section{Implications for urban green management}

This study has some implications for urban planning and urban green space design. As surface temperature is an upward radiation flux that is captured by remote sensors, it has the potential to radiate and conduct a cooler flux to the surrounding air and neighboring objects. Larger green patches result in cooler UCIs, either because less heat flux from built up areas is emitted to the core area of the green patch, especially for patches of waters and trees, or because more of the cool flux is conducted to the surroundings. Effects of shape metrics on UCI vary during the seasons, which can represent different climate zones. This finding embodies some hints for urban green management for cities with different climates, such as cold and dry climate or warm and humid climate.

First, patch area (PA) was found to affect UCIs in all seasons, especially strongly in summer, and for all green types, similar to the results of other observational studies (Li et al., 2012; Onishi et al., 2010; Susca et al., 2011). PA also affected the UCI more strongly than shape metrics in summer. Generally, UCI effects are more important in warm season for most cities. Therefore, this finding justifies better management of urban green spaces to increase tree canopies and the greening of roofs, parking lots, and even building walls to add green cover. This finding also suggests urban green managers in areas with long summer time that more efforts be put to urban green area than to green shape.

Second, for areas with warm and humid climates, water bodies and forests are more suggested than are shrubs and grass for urban green infrastructures, as correlation analysis shows stronger correlation coefficients between their pattern metrics and their UCIs, especially in warm dates. This implies that a small change of the pattern of waters or trees will result in cooler islands in warm periods.
Thirdly, patch shape also matters in the formation of UCIs according to the different indicator values of Trees, River, and Crops, especially in dry and cool periods. Thus, urban green design in areas with dry and cool climates could particularly consider the shape of these green types.

\section{Implications for methodology}

Firstly, this study explored the relation between UCIs and urban green pattern metrics, which were easily determined from the existing landscape metrics software (Patch Analyst of Rempel et al. (2012) or FRAGSTATS of McGarigal et al. (2012)). Connectivity for vector-format patches was difficult to derive with landscape software but could be easily analyzed with $\operatorname{ArcGIS}^{\mathrm{TM}}$ (Cao et al., 2010; Sun et al., 2012).

Secondly, the metrics used in this study are easier to implement in urban planning. For instance, the metrics are better than the NDVI, which is an urban green surface metric/parameter (McGarigal et al., 2009) that is hard to utilize in implementation of green planning. Area and shape, as characteristics of green patches, are the elementary factors in arranging the patterns of urban land cover. Although NDVI has been proved to correlate negatively with LST (Weng and Lu, 2008), it is a parameter for the management of urban green spaces, and is secondary to patch spatial features in urban green planning.

Finally, Spearman's rho was believed to be more reasonable than Pearson's and was used for bivariate correlation analysis. This was because the landscape metrics used here were developed for categorical map patterns, thus, the normal distribution of these derived metrics could not be guaranteed. Pearson's rho could result in different conclusions, as shown Table 6. For example, SI of water bodies showed no effect on UCIs of water bodies, which would be less reasonable.

\section{Pattern metrics as predictors of UCIk}

We found in our studies that SI and FD as shape metrics were valuable for UCI prediction, whereas PAR was not. The indicator value of SI reveals that with, a certain patch area, a patch with convoluted or "plane-filling" edge results in lower minimum temperature than circular patch, and thus forms a cooler island. This is consistent with previous findings from both the studies on parks (e.g., Cao et al., 2010) and the studies on waters in Beijing (Sun et al., 2012; Sun and Chen, 2012) that found complex shape patches bring more cooling effect. PAR as a shape metric, however, has been proved unsuitable for analyzing the effects of pattern on UCIs. PAR increases if we increase the patch perimeter while holding its area constant, such that the patch shape grows complex, and it should have correlated negatively with UCI as did SI or FD. This is contrary to the effect of metrics such as SI or FD. The vagueness of PAR is caused by the problem of PAR itself. Although it is still used in some studies (Hassett et al., 2012; McGarigal et al., 2012), PAR changes with the change in patch size. For example, two quadrates with the same shape and with edge lengths of 2 and 8 meters result in PARs of 2 and $1 / 2$, respectively. This proves it to be a problematic metric.

It is also inappropriate to treat all green space types in a general manner to determine a predictive indicator. Witness that FD correlated with UCIs of different dates and in different direction between vegetation and waters was actually attributed to the effect of FD on UCIs varying abruptly among these types. In addition, shape, edge and connectivity metrics performed well in bivariate correlation analysis; however, the regression results revealed that only FD was better than PA or connectivity metrics for predicting Trees and Crops temperatures. Shape metrics performed well in bivariate correlation analysis for Rivers and Lakes, whereas in stepwise regression, only FD was selected as the indicating metric for Rivers. 
Table 6

Pearson correlation coefficients among urban green pattern metrics and UCI of the four dates.

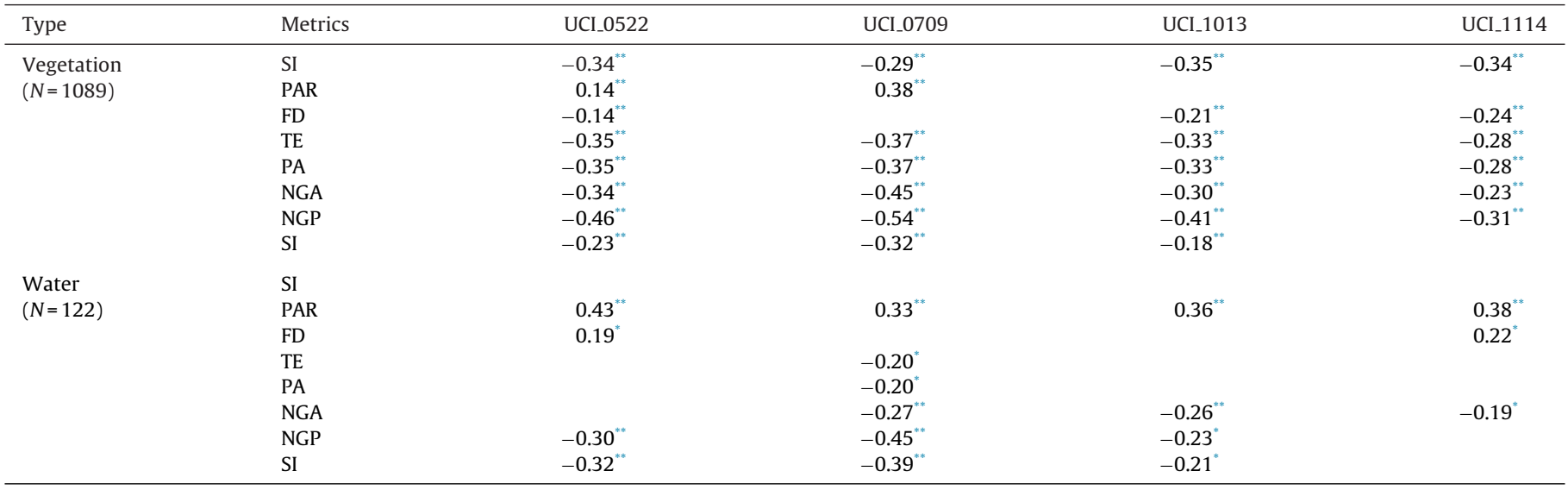

\footnotetext{
* Correlation is significant at the 0.05 level (2-tailed).

** Correlation is significant at the 0.01 level (2-tailed).

The digits $0522,0709,1013$, and 111 here represent the dates May 22, July 9, October 13 , and November $14 . N$ is the number of samples.
}

Therefore, not only vegetation but also waters should be treated specifically and differently.

There are some differences in the indicator values between our results and those of previous studies. In our study, PA correlated negatively with UCI, indisputably indicating that more green area will result in cooler UCI, similar to the findings reported in other landscape level studies (Liu and Weng, 2008; Buyantuyev and Wu, 2010; Li et al., 2011). What is different is that shape metrics performed better in our regression models than in bivariate correlation when combining PA with shape metrics, whereas the previous studies stated that the percentage of green landscape (PLAND in their studies but PA here) is more important in deciding LST than other metrics. This is because previous studies have been conducted at the landscape level and vegetation was treated as a whole, and so were waters. Our results showed that River and Lake had different cooling effects and formed different cooling islands, similar to the results of courtyard observation (Shashua-Bar et al., 2009) and urban-scale water/land research studies (Sun et al., 2012).

In general, the indicative metrics are different for the six green types. It is inappropriate to generalize predictors for UCI because the physical properties of different green types are different. Therefore, a combination of urban green types and pattern metrics as well as partial correlation analysis among metrics are prerequisites for analysis of the effect of patterns on UCIs.

\section{Limitations and further study}

This study has several limitations. The first is the representation of seasonal UCIs. We used only the minimum LST for UCI. We have reasons for this: the maximum and mean LST may be affected by certain concrete built-up areas within a patch. However, the mean LST promised by pure green pixels and the median LST could be tested to determine the pattern effects on the LST range of a whole green patch in future studies. We also used LST on one date to represent a season because daily or monthly good quality Landsat images were not available. Therefore, true winter UCI were actually lacked.

The second limitation is the connectivity analysis. Our buffer analysis for connectivity used only one distance ( $50 \mathrm{~m}$ ). More distances, such as $10 \mathrm{~m}$ and $20 \mathrm{~m}$, could be tested to find the optimal buffer distances of the neighborhood affecting the UCI. Thirdly, higher resolution remote sensed images and auxiliary information that shows the detail of urban green spaces, such as growing conditions of vegetation, tree species, and depth of water in lakes/rivers could have been considered in addition to urban green pattern metrics.

Finally, combination of other factors, including weather conditions such as air temperature/humidity and wind velocity, the sky view factor (Ng et al., 2012), street orientation (Oliveira et al., 2011) and even socio-economic data (Buyantuyev and Wu, 2010; Huang et al., 2011) into the analysis would make our study to be better synthesized with other research, and would be a good target for future studies.

\section{Conclusion}

This study investigated seasonal and type variations in the effects of urban green spatial patterns on surface UCIs of rapidly urbanized Beijing. Linking the methods of landscape ecology, the geophysical information system and remote sensing, the results show that not only patch size but also its shape, edge or connectivity has effects on UCIs. The effects vary considerably among the seasons and green types. Consequently, redesign of urban green shape, proper management of existing urban green as well as increasing urban green area are all effectible measures to cool the city. Water and woodland are suggested in urban planning concerning cooling effects, especially in summer. In the context of rapid urbanization and global climate change, local landscape adaptation through regulation and management of urban green patterns is critical, but the suggestions for adaptation need to be based on science. This study improves our understanding of how landscape patterns affect environmental processes with respect to UCIs, how pattern metrics perform in predicting UCIs and at the same time leaves some room for further studies on microclimate regulation.

\section{Acknowledgements}

The authors own great thanks to the two anonymous reviewers for their time and comments. The Landsat ETM+ images are in courtesy of the U.S. Geological Survey. The whole work was funded by the National Natural Science Foundation of China (41230633) and the Innovation Project of State Key Laboratory of Urban and Regional Ecology of China (SKLURE2008-1-02).

\section{Appendix A. Supplementary data}

Supplementary data associated with this article can be found, in the online version, at http://dx.doi.org/10.1016/j.ufug.2014.07.006. 


\section{References}

Armson, D., Stringer, P., Ennos, A.R., 2012. The effect of tree shade and grass on surface and globe temperatures in an urban area. Urban For. Urban Green. 11 (3), 245-255.

Benedict, M.A., McMahon, E.T., 2006. Green Infrastructure: Linking Landscapes and Communities. Island Press.

Bruse, M., Fleer, H., 1998. Simulating surface-plant-air interactions inside urban environments with a three dimensional numerical model. Environ. Model. Softw. 13 (3), 373-384.

Buyantuyev, A., Wu, J., 2010. Urban heat islands and landscape heterogeneity: linking spatiotemporal variations in surface temperatures to land-cover and socioeconomic patterns. Landsc. Ecol. 25 (1), 17-33.

Cao, X., Onishi, A., Chen, J., Imura, H., 2010. Quantifying the cool island intensity of urban parks using ASTER and IKONOS data. Landsc. Urban Plan. 96 (4), 224-231.

Chander, G., Groeneveld, D., 2009. Intra-annual NDVI validation of the Landsat 5 TM radiometric calibration. Int. J. Remote Sens. 30 (6), 1621-1628.

Chen, L., Tian, H., Fu, B., Zhao, X., 2009. Development of a new index for integrating landscape patterns with ecological processes at watershed scale. Chin. Geogr. Sci. 19 (1), 37-45.

Cohen, P., Potchter, O., Matzarakis, A., 2012. Daily and seasonal climatic conditions of green urban open spaces in the Mediterranean climate and their impact on human comfort. Build. Environ. 51, 285-295

Fischer, E., Seneviratne, S., Lüthi, D., Schär, C., 2007. Contribution of land-atmosphere coupling to recent European summer heat waves. Geophys. Res. Lett. 34, L06707.

Frumkin, H., McMichael, A., 2008. Climate change and public health thinking, communicating, acting. Am. J. Prev. Med. 35 (5), 403-410.

Hamada, S., Ohta, T., 2010. Seasonal variations in the cooling effect of urban green areas on surrounding urban areas. Urban For. Urban Green. 9 (1), 15-24.

Harnik, P., 2010. Urban Green: Innovative Parks for Resurgent Cities. Island Press, Washington, DC

Hassett, E.M., Stehman, S.V., Wickham, J.D., 2012. Estimating landscape pattern metrics from a sample of land cover. Landsc. Ecol. 27 (1), 133-149.

Heidt, V., Neef, M., 2008. Benefits of urban green space for improving urban climate. In: Carreiro, M., Song, Y.-C., Wu, J. (Eds.), Ecology, Planning, and Management of Urban Forests. Springer, New York, pp. 84-96.

Huang, G., Zhou, W., Cadenasso, M.L., 2011. Is everyone hot in the city? Spatial pattern of land surface temperatures, land cover and neighborhood socioeconomic characteristics in Baltimore, MD. J. Environ. Manage. 92 (7), 1753-1759.

Kantzioura, A., Kosmopoulos, P., Zoras, S., 2012. Urban surface temperature and microclimate measurements in Thessaloniki. Energy Build. 44, 63-72.

Li, J., Song, C., Cao, L., Zhu, F., Meng, X., Wu, J., 2011. Impacts of landscape structure on surface urban heat islands: a case study of Shanghai, China. Remote Sens. Environ. 115 (12), 3249-3263.

Li, X., Zhou, W., Ouyang, Z., Xu, W., Zheng, H., 2012. Spatial pattern of greenspace affects land surface temperature: evidence from the heavily urbanized Beijing metropolitan area, China. Landsc. Ecol. 27 (6), 887-898.

Liu, H., Weng, Q, 2008. Seasonal variations in the relationship between landscape pattern and land surface temperature in Indianapolis, USA. Environ. Monit. Assess. 144 (1), 199-219.

Mackey, C.W., Lee, X., Smith, R.B., 2012. Remotely sensing the cooling effects of city scale efforts to reduce urban heat island. Build. Environ. 49, 348-358.

Matzarakis, A., Rutz, F., Mayer, H., 2010. Modelling radiation fluxes in simple and complex environments: basics of the RayMan model. Int. J. Biometeorol. 54 (2), 131-139.
McGarigal, K., Tagil, S., Cushman, S., 2009. Surface metrics: an alternative to patch metrics for the quantification of landscape structure. Landsc. Ecol. 24 (3) 433-450.

McGarigal, K., Cushman, S., Ene, E., 2012. FRAGSTATS v4: Spatial Pattern Analysis Program for Categorical and Continuous Maps. Computer software program produced by the authors at the University of Massachusetts, Amherst. http://www.umass.edu/landeco/research/fragstats/fragstats.html

NASA, 2000. Landsat 7 science data users' handbook, PP.101. http://landsathandbook.gsfc.nasa.gov/

Ng, E., Chen, L., Wang, Y., Yuan, C., 2012. A study on the cooling effects of greening in a high-density city: an experience from Hong Kong. Build. Environ. 47, 256-271.

Oliveira, S., Andrade, H., Vaz, T., 2011. The cooling effect of green spaces as a contribution to the mitigation of urban heat: a case study in Lisbon. Build. Environ. 46 (11), 2186-2194.

Onishi, A., Cao, X., Ito, T., Shi, F., Imura, H., 2010. Evaluating the potential for urban heat-island mitigation by greening parking lots. Urban For. Urban Green. 9 (4), 323-332.

Opdam, P., Luque, S., Jones, K.B., 2009. Changing landscapes to accommodate for climate change impacts: a call for landscape ecology. Landsc. Ecol. 24 (6), 715-721.

Rempel, R.S., Kaukinen, D., Carr, A.P., 2012. Patch Analyst and Patch Grid Patch Analyst Help. Ontario Ministry of Natural Resources http://www.cnfer. on.ca/SEP/patchanalyst/

Schwarz, N., Lautenbach, S., Seppelt, R., 2011. Exploring indicators for quantifying surface urban heat islands of European cities with MODIS land surface temperatures. Remote Sens. Environ. 115 (12), 3175-3186.

Shashua-Bar, L., Pearlmutter, D., Erell, E., 2009. The cooling efficiency of urban landscape strategies in a hot dry climate. Landsc. Urban Plan. 92 (3-4), 179-186.

Sobrino, J., Raissouni, N., 2000. Toward remote sensing methods for land cover dynamic monitoring: application to Morocco. Int. J. Remote Sens. 21 (2) 353-366.

Sobrino, J., Jimenez-Munoz, J., Paolini, L., 2004. Land surface temperature retrieval from LANDSAT TM 5. Remote Sens. Environ. 90 (4), 434-440.

Sun, R., Chen, A., Chen, L., Lü, Y., 2012. Cooling effects of wetlands in an urban region: the case of Beijing. Ecol. Indic. 20, 57-64.

Sun, R., Chen, L., 2012. How can urban water bodies be designed for climate adaptation? Landsc. Urban Plan. 105 (1-2), 27-33.

Susca, T., Gaffin, S., Dell'Osso, G., 2011. Positive effects of vegetation: urban heat island and green roofs. Environ. Pollut. 159 (8-9), 2119-2126.

Voogt, J.A., Oke, T.R., 2003. Thermal remote sensing of urban climates. Remote Sens. Environ. 86 (3), 370-384

Wang, J., Wang, K., Wang, P., 2007. Urban heat (or cool) island over Beijing from MODIS land surface temperature. J. Remote Sens. 11 (3), 330 (In Chinese with English Abstract)

Weng, Q., Lu, D., 2008. A sub-pixel analysis of urbanization effect on land surface temperature and its interplay with impervious surface and vegetation coverage in Indianapolis, United States. Int. J. Appl. Earth Obs. Geoinf. 10 (1), 68-83.

Weng, Q., 2009. Thermal infrared remote sensing for urban climate and environmental studies: methods, applications, and trends. ISPRS J. Photogramm. Remote Sens. 64 (4), 335-344.

Zhou, W., Huang, G., Cadenasso, M.L., 2011. Does spatial configuration matter? Understanding the effects of land cover pattern on land surface temperature in urban landscapes. Landsc. Urban Plan. 102 (1), 54-63.

Zoulia, I., Santamouris, M., Dimoudi, A., 2009. Monitoring the effect of urban green areas on the heat island in Athens? Environ. Monit. Assess. 156 (1-4), 275-292. 